\title{
Efeitos de baixas doses de cloprostenol via intramuscular ou submucosavulvar na indução do estro e taxas de prenhez em vacas Nelore
}

\section{Effects of lower doses of cloprostenol intramuscular or into vulvar submucosa on estrus induction and pregnancy rates in Nelore cows}

\author{
Marcelo George Mungai Chacur ${ }^{1 *}$; Paulo Tiago Ferreira Aurélioº; Orivaldo Scalon \\ Júnior $^{3}$; Leandro Inague ${ }^{3}$; Luis Fernando Scalon ${ }^{4}$; Sérgio do Nascimento Kronka ${ }^{5}$
}

\section{Resumo}

\begin{abstract}
O presente estudo teve o objetivo de comparar os efeitos de baixas doses de cloprostenol na via intramuscular (IM) ou submucosavulvar (SMV) na detecção do estro e taxas de prenhez em vacas Nelore. Utilizou-se 100 vacas Nelore cíclicas com condição de escore corporal (CEC 3,5) na escala de 1 a 5 (Radostitis; Blood, 1986) com 170 \pm 11 dias pós-parto. As fêmeas foram divididas aleatoriamente em cinco grupos ( $\mathrm{N}=20)$ G1 a G5 e tratadas com cloprostenol (Ciosin $®)$ no dia zero (D0) e no dia 11

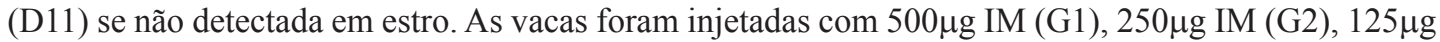

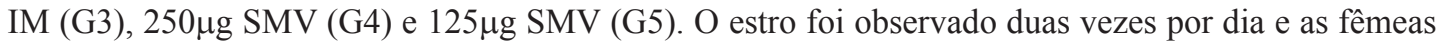
inseminadas artificialmente 12 horas após a detecção do mesmo. Não houve diferença estatística entre grupos ( $\mathrm{P}>0,80)$ na indução do estro (intervalo em horas: primeira injeção - estro): 16/20 - 96,00 h (G1), 13/20 - 90,42 h (G2), 10/20 - 84,45 h (G3), 15/20 - 87,86 h (G4), 12/20 - 81,25 h (G5) e para a segunda injeção (P>0,10): 4/20 - 67,50 h (G1), 7/20 - 85,50 h (G2), 10/20 - 57,00 h (G3), 5/20 - 70,60 h (G4), $8 / 20-60,00 \mathrm{~h}(\mathrm{G} 5)$. Não houve diferença significativa $\left(0,65^{\text {ns }}\right)$ entre grupos para as taxas de prenhez: $40 \%$ (G1), 45\% (G2), 50\% (G3), 40\% (G4) e 40\% (G5). Os resultados demonstram que os tratamentos com baixas doses de cloprostenol intramuscular ou submucosavulvar podem ser usados na indução do estro, com taxas de prenhez semelhantes em vacas Nelore cíclicas, com boa condição corporal.

Palavras-chave: Vacas zebu, Bos taurus indicus, prostaglandina, microdoses, inseminação artificial
\end{abstract}

\begin{abstract}
The present study aims to compare the effects of lower doses of cloprostenol intramuscular (IM) or into vulvar submucosa (IVS) on estrus induction and pregnancy rate in Nelore cows. A total of 100 cycling Nelore cows with body condition score (BCS 3.5) 1 to 5 scale (Radostitis; Blood, 1986) and $170 \pm 11$ days postpartum. Females were randomly divided in 5 groups $(\mathrm{N}=20) \mathrm{G} 1$ to $\mathrm{G} 5$ and treated with cloprostenol (Ciosin $($ ) on day 0 (D0) and on day 11 (D11) if not detected in estrus. Cows were injected

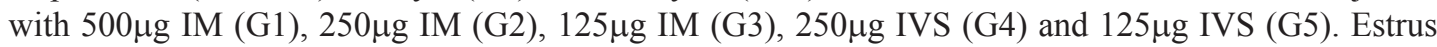
was observed twice a day and the females artificially inseminated 12 hours after heat detection. There was no statistical difference $(\mathrm{P}>0.80)$ between groups in the estrus induction (first injection to estrus interval): $16 / 20$ - 96.00 hours (G1), 13/20 - 90.42 hours (G2), 10/20 - 84.45 hours (G3), 15/20 - 87.86
\end{abstract}

\footnotetext{
Médico Veterinário - Prof. Dr. Universidade do Oeste Paulista - UNOESTE - Presidente Prudente-SP. E-mail: chacur@unoeste.br

Médico Veterinário - Mestre em Ciência Animal pela UNOESTE.

Médicos Veterinários - Ex-alunos de Iniciação Científica da UNOESTE.

4 Médico Veterinário autônomo.

5 Engenheiro Agrônomo - Prof. Dr. Faculdade de Ciências Agrárias e Veterinárias de Jaboticabal - UNESP.

Autor para correspondência
} 
hours (G4), 12/20 - 81.25 hours (G5) and second injection (P>0.10): 4/20 - 67.50 hours (G1), 7/20 85.50 hours (G2), 10/20 - 57.00 hours (G3), 5/20 - 70.60 hours (G4), 8/20 - 60.00 hours (G5). There was no statistical difference $\left(0.65^{\text {ns }}\right)$ between groups in the pregnancy rates: $40 \%(\mathrm{G} 1), 45 \%(\mathrm{G} 2), 50 \%$ (G3), 40\% (G4), 40\% (G5). The results demonstrate that the treatments with lower doses of cloprostenol intramuscular or into vulvar submucosa may be used to induce heat with similar pregnancy rates in cycling Nelore cows with good body condition.

Key words: Zebu cows, Bos taurus indicus, prostaglandin, micro doses, artificial insemination

\section{Introdução}

Nafaseluteínica ocorpolúteoproduzprogesterona em quantidades crescentes do quarto ao décimo dia do ciclo estral, e a secreção se mantém estável até que ocorra a luteólise, em torno do décimo quinto ao vigéssimo dia (LUQUE; HUTTER; MONTES, 1983; HAFEZ; HAFEZ, 2004).

A relação do útero com o ovário no controle da regressão do corpo lúteo foi primeiramente descrita por Loeb (1923, 1927), sendo que a secreção em pulsos da prostaglandina pelo útero coincidiu com a queda da progesterona, durante a luteólise (KINDAHL et al., 1984).

A íntima relação entre a veia uterina e a artéria ovariana, melhor observada em ruminantes e na porca, facilita a transferência de prostaglandina produzida no endométrio para o ovário (GINTHER; DEL CAMPO, 1974). Apesar da estreita ligação anatômica entre os órgãos da genitália interna e externa e por tratar-se de uma região altamente irrigada, não se sabe se existem anastomoses entre os vasos dos órgãos internos e externos (SCHALLER, 1993).

Existe grande variabilidade nas respostas aos tratamentos com prostaglandina, no que se refere ao intervalo tratamento-estro (KASTELIC; KNOPF; GINTHER, 1990; SAVIO; BOLAND; ROCHE, 1990). Essa variabilidade no intervalo entre a administração de PGF-2 $\propto$ e a manifestação do estro se deve a existência de um padrão contínuo de crescimento e atresia dos folículos ovarianos durante o ciclo estral (LUCY et al., 1992).

Os animais que apresentam folículos dominantes em fase de crescimento, durante o momento de administração de PGF- $2 \propto$, manifestam estro 48 a 60 horas após o uso do agente luteolítico, enquanto aqueles que possuem folículos em fase estática ou de regressão levam cerca de 5 a 7 dias (BO et al., 1994).

Trabalhos demonstraram a utilização de doses reduzidas de PGF-2 $\propto$ por via submucosa vulvar ou intra-uterina para induzir luteólise de forma similar a PGF-2 $\propto$ administrada por via intramuscular (ONO et al., 1982; CHAUHAN et al., 1986; GINTHER, 1981).

A otimização do manejo reprodutivo pode ser alcançado mediante a concentração do período de manifestação do estro (VALLE, 1991). Em bovinos de corte, o uso de drogas luteolíticas tem aumentado nos últimos anos, devido à necessidade de concentrar a época de cobertura, bem como de maximizar a mão-de-obra da fazenda na época de parição dos animais (PESSÔA, 2003).

Este estudo teve como objetivo avaliar o efeito do cloprostenol em diferentes doses nas vias intramuscular e submucosa vulvar nas taxas de indução do estro e de prenhez em vacas Nelore.

\section{Material e Métodos}

Animais e local do experimento

O presente experimento foi realizado no estado do Mato Grosso do Sul, Brasil, nos meses de abril a junho de 2007. Foram utilizadas 100 vacas da raça Nelore, com idades de quatro a seis anos, com escore corporal 3,5 na escala de 1 a 5 descrita por Radostitis e Blood (1986), criadas em pastagens de Brachiaria decumbens, com sal mineral e água "ad 
libitum". As vacas foram examinadas pela palpação retal e exame ultrassonográfico, com a utilização de aparelho da marca Pie Medical® Scanner 480, para verificar a ausência de prenhez e a integridade anatômica do aparelho reprodutor interno. As vacas apresentavam $170 \pm 11$ dias de período pós-parto.

\section{Tratamentos}

As 100 vacas foram divididas aleatoriamente em cinco grupos experimentais, com 20 animais cada, da seguinte forma: grupo 1 (G1): $2 \mathrm{~mL}-500 \mu \mathrm{g}$ de D-cloprostenol sódico (Ciosin $\AA$ ) intramuscular (IM). grupo 2 (G2): $1 \mathrm{~mL}-250 \mu \mathrm{g}$ IM, grupo

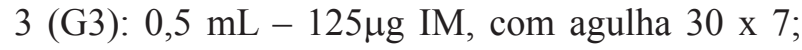
grupo 4 (G4): $1 \mathrm{~mL}-250 \mu \mathrm{g}$ na submucosa vulvar (SMV) e grupo 5 (G5): $125 \mu \mathrm{g}$ SMV, com agulha fina $(13 \times 4,5)$. Todas as vacas foram mantidas sob observação de cio, juntamente com 5 rufiões com buçal marcador, durante 5 dias. Nas vacas que não apresentaram cio, após observação por 5 dias, uma segunda aplicação de cloprostenol foi efetuada 11 dias após a primeira, nas mesmas doses e vias.

A observação do cio foi realizada duas vezes ao dia, no período da manhã (6:30 às 7:30 h) e à tarde (17:30 às 18:30 h), por meio da marcação de tinta deixada pelos rufiões e pela visualização do comportamento dos animais como a presença de muco na vulva, inquietação e comportamento de monta. As inseminações artificiais foram efetuadas aproximadamente 12 horas após a constatação do estro.

A partida de sêmen utilizada foi avaliada, apresentando motilidade progressiva de $50 \%$, vigor 3, concentração de 23,9 × $10^{6}$ espermatozóides viáveis e morfologia espermática com $86 \%$ de espermatozóides normais, segundo normas do Colégio Brasileiro de Reprodução Animal (1998).

O diagnóstico de prenhez foi realizado 50 dias após o término da última inseminação, por meio de palpação retal e confirmada com o exame de ultrasonografia transretal com transdutor de $5 \mathrm{MHz}$.

\section{Análise estatística}

Utilizou-se delineamento inteiramente casualizado com cinco grupos (G1, G2, G3, G4 e G5) com 20 repetições para cada grupo. Os dados foram submetidos à análise de variância pelo teste $\mathrm{F}$ (BANZATTO; KRONKA, 2006).

O modelo matemático utilizado foi:

$$
\mathrm{Y}_{\mathrm{ij}}=\mathrm{m}+\mathrm{g}_{\mathrm{i}}+\mathrm{e}_{\mathrm{ij}}
$$

onde,

$\mathrm{y}_{\mathrm{ij}}=$ valor observado no animal do grupo $\mathrm{i}$, na repetição j

$\mathrm{m}=$ média geral

$\mathrm{g}_{\mathrm{i}}=$ efeitodo grupo $\mathrm{i}$

$\mathrm{e}_{\mathrm{ij}}=$ efeito do acaso no animal do grupo $\mathrm{i}$, na repetição j

Para o estudo da prenhez na comparação entre grupos, utilizou-se a metodologia do Qui-Quadrado $\left(\mathrm{X}_{\mathrm{y}}^{2}=0,65^{\mathrm{ns}}\right)$.

\section{Resultados e Discussão}

Nas décadas de 80 e 90, autores relataram o emprego de baixas doses de prostaglandina com resultados satisfatórios na indução do estro, principalmente, em raças taurinas. Em vacas zebuínas existe a necessidade de se explorar melhor os resultados perante as baixas doses de prostaglandina. O uso da via submucosa vulvar para a deposição do agente luteolítico em menores doses, está relacionado à hipótese de que a resposta fisiológica possa ser boa, pois o tecido da injeção está mais próximo do ovário, em relação à via intramuscular. Schaller (1993) relatou que a despeito de se tratar de uma região altamente irrigada, não se sabe se existem anastomoses entre os vasos dos órgãos do aparelho reprodutor interno e externo

As percentagens de vacas que apresentaram estro com a primeira dose de cloprostenol variaram de $50 \%(10 / 20)$ no grupo G3 a $80 \%(16 / 20)$ no grupo 
G1 e na segunda dose entre 25\% (5/20) no G1 e $50 \%(10 / 20)$ no G3, totalizando $100 \%$ das fêmeas que responderam à medicação para os 5 grupos (Tabela 1). Resultado esse do grupo G1, superior à manifestação de estro de $50 \%$, em vacas da raça Nelore, com dose de $500 \mu \mathrm{g}$ via intramuscular (CASTILHO; DAYAN; BARROS, 1997;
FIGUEIREDO et al., 1997). Após tratamentos com prostaglandina, na presença de corpo lúteo funcional (PINHEIRO et al., 1998). Em vacas repetidoras de cio, Nelore x Chianina, índices de indução do estro entre $56 \%$ e $79,16 \%$ foram relatadas, com o uso de cloprostenol (CHACUR et al., 2005).

Tabela 1. Intervalo em horas entre a administração intramuscular (IM) ou na submucosa vulvar (SMV) de cloprostenol para a detecção do estro após a 1 $1^{\mathrm{a}}$ dose (dia zero - D0) ou 2 dose (dia onze - D11), em vacas Nelores.

\begin{tabular}{|c|c|c|c|}
\hline Grupos & $\mathbf{N}$ & $\begin{array}{c}1^{\text {a dose (horas) }} \\
\text { D0 }\end{array}$ & $\begin{array}{c}2^{\mathrm{a}} \text { dose (horas) } \\
\text { D11 }\end{array}$ \\
\hline 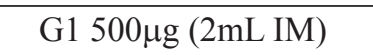 & 20 & $16(96,00) \mathrm{a}$ & $4(67,50) \mathrm{a}$ \\
\hline 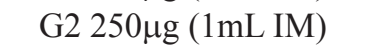 & 20 & $13(90,42)$ a & $7(85,50) \mathrm{a}$ \\
\hline G3 $125 \mu \mathrm{g}(0,5 \mathrm{~mL}$ IM $)$ & 20 & $10(84,45)$ a & $10(57,00)$ a \\
\hline 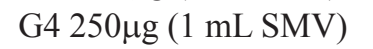 & 20 & $15(87,86)$ a & $5(70,60) a$ \\
\hline G5 $125 \mu \mathrm{g}(0,5 \mathrm{~mL}$ SMV $)$ & 20 & $12(81,25) \mathrm{a}$ & $8(60,00) \mathrm{a}$ \\
\hline Teste F & & $\begin{array}{c}0,41^{\mathrm{NS}} \\
(\mathrm{P}>0,80)\end{array}$ & $\begin{array}{c}2,16^{\mathrm{NS}} \\
(\mathrm{P}>0,10)\end{array}$ \\
\hline Desvio padrão & & 33,46 & 21,73 \\
\hline C.V. $(\%)$ & & 38,02 & 31,90 \\
\hline
\end{tabular}

NS - não significativo $(\mathrm{P}>0,05)$.

Nos grupos G1 e G4, para a primeira dose, as taxas de indução do estro foram semelhantes às relatadas em vacas de raças européias, entre $70 \%$ e $90 \%$ (TANABE; HANN, 1984) e compreendido dentro do intervalo de $66 \%$ a $97 \%$ segundo Laverdiere et al. (1995).

Com uma única aplicação de prostaglandina, $80 \%$ das vacas manifestaram estro no grupo G1 e $75 \%$ no grupo G4 (Tabela 1), semelhante ao relato no Brasil por Fernandes, Torres e Costa (1994) obtendo taxas de manifestação de estro em

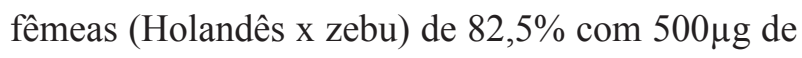
cloprostenol intramuscular.

A taxa máxima de indução de estro foi de $80 \%$ (G1) com uma dose de cloprostenol, até 20\% (G4) na segunda dose; superior aos $30 \%$ e inferior aos $40 \%$, respectivamente, descritos por Galina, Orihuela e Duchateau (1987), com os mesmos protocolos hormonais.
Obteve-se $75 \%$ de indução de estro no grupo G4 na primeira dose, superando o índice de $40 \%$ relatado por Pinheiro et al. (1998), quando do uso do mesmo tratamento. O grupo G5, demonstrou $60 \%$ de taxa de indução de estro, inferior às observadas por Laverdiere et al. (1995) com 74,7\% em vacas Shortorn-Hereford e Alvarez et al. (1991) com 88\% (8/9) em novilhas cruzadas (zebuíno x taurino) tratadas com $250 \mu \mathrm{g}$ ou $125 \mu \mathrm{g}$ de cloprostenol via submucosa vulvar. Sugere-se que em vacas Nelores, o uso da via submucosa vulvar em pequenas doses não apresente a mesma eficácia em relação às fêmeas de origem mestiça ou taurina.

A aplicação de cloprostenol resultou em 100\% de indução do estro nos 5 grupos experimentais, com o uso de duas injeções com intervalo de 11 dias, revelando resposta altamente satisfatória. Resposta essa que pode estar associada aos seguintes fatores intrínsicos às vacas: 1) escore de condição corporal de 3,5 (1 a 5) com balanço energético positivo; 2) 
involução uterina completa e 3) atividade ovariana luteal cíclica presente, proporcionando condições anatômicas e endócrinas desejáveis para a resposta fisiológica perante a inoculação do hormônio. Dessa forma, sugere-se que os resultados similares com baixas doses e diferentes vias de aplicação, para as taxas de prenhez, possam estar associados a esses três fatores.

Outros pontos importantes que podem estar relacionados com a alta manifestação dos estros são: 1) a seleção das vacas por meio de avaliação ginecológica e 2) a eficiência do manejo de detecção de estros. Supõe-se que as fêmeas Nelores necessitem de doses inferiores às doses clássicas para raças taurinas, tendo em vista os resultados obtidos.

$\mathrm{Na}$ avaliação do intervalo em horas entre a administração intramuscular ou na submucosa vulvar de cloprostenol para a manifestação do cio, não houve diferença significativa entre grupos para a primeira $(\mathrm{P}>0,80)$ ou segunda dose $(\mathrm{P}>0,10)$, conforme a Tabela 1. Observa-se na primeira dose que as vacas do grupo G5 apresentaram o menor período para a manifestação do estro com 81,25 h, sendo o maior intervalo no G1 com 96 h. Com relação à segunda dose, o intervalo esteve entre 57 h no grupo G3 e 85,5 h no grupo G2.

Tempos médios esses, compreendidos entre o intervalo relatado por outros pesquisadores, onde a receptividade à monta se fez presente entre 48 a 120 horas após a injeção de análogos de prostaglandina, acima de 120 horas o estro pode ocorrer de forma natural e não devido à indução pelo produto administrado (WRIGHT; MALMO, 1992).
Uma das limitações da utilização de PGF-2œ é a baixa taxa de sincronização na indução do estro (TANABE; HANN, 1984). A variabilidade no intervalo entre a administração de PGF-2 $\propto$ e a manifestação do estro se deve a existência de um padrão contínuo de crescimento e atresia dos folículos ovarianos durante o ciclo estral (LUCY et al., 1992). Do ponto de vista biológico, houve um menor intervalo em horas no grupo G5 em relação ao G4, na administração de cloprostenol na submucosa vulvar e o aparecimento dos sinais do estro.

Nos grupos experimentais, as médias em horas desde a aplicação do cloprostenol até a manifestação do estro nas vacas Nelores, foram inferiores às descritas em outras raças zebuínas por Borges et al. (2003) que obtiveram $113,1 \mathrm{~h}$ a $119,5 \mathrm{~h}$ em vacas Gir e Nelore; Moreno et al. (1986) com 114 h na raça Indubrasil e Alves (1999) com médias de 93 h e 97 h para as raças Gir e Guzerá, respectivamente.

Sugere-se que a grande variabilidade no intervalo entre a injeção de prostaglandina e a manifestação do estro nas vacas do presente estudo, esteja relacionada à teoria de Bo et al. (1994), onde os animais que apresentam folículos dominantes em fase de crescimento, durante o momento de administração de PGF-2 $\propto$, manifestam estro 48 a 60 horas após o uso do agente luteolítico, enquanto aqueles que possuem folículos em fase estática ou de regressão levam cerca de 5 a 7 dias.

Para o total de vacas prenhes, não houve diferença significativa $\left(0,65^{\text {ns }}\right)$ entre os grupos, com taxas de $40 \%$ para os grupos G1, G4 e G5; de $45 \%$ no grupo G2 e de 50\% para o grupo G3 (Tabela 2). 
Tabela 2. Taxas de prenhez de vacas Nelore submetidas a diferentes tratamentos com cloprostenol, administrado nas vias intramuscular (IM) ou submucosa vulvar (SMV).

\begin{tabular}{|c|c|c|c|}
\hline Grupos & $\mathbf{N}$ & Prenhes (N) & $\%$ \\
\hline 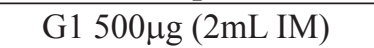 & 20 & 8 & 40 \\
\hline 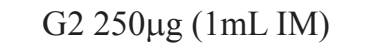 & 20 & 9 & 45 \\
\hline G3 $125 \mu \mathrm{g}(0,5 \mathrm{~mL} \mathrm{IM})$ & 20 & 10 & 50 \\
\hline 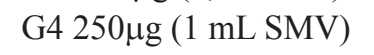 & 20 & 8 & 40 \\
\hline G5 $125 \mu \mathrm{g}(0,5 \mathrm{~mL} \mathrm{SMV})$ & 20 & 8 & 40 \\
\hline total & 100 & 43 & 43 \\
\hline
\end{tabular}

$\mathrm{X}_{\mathrm{y}}^{2}=0,65^{\text {ns }}$

Resultados esses superiores aos dos estudos com uso de cloprostenol, descritos na raça Nelore, obtendo $15 \%$ de prenhez em vacas com escore corporal 2 (1 a 5), na época das secas (CHACUR et al., 2000) e de $40 \%$ de prenhez em vacas Nelore $\mathrm{x}$ Chianina repetidoras de cio (CHACUR et al., 2005). Por outro lado, similares aos relatos de Pessôa (2003) na época das águas em vacas Nelores, relatando eficiência similar frente à indução do estro com luprostiol na dose de $500 \mu \mathrm{g}$ intramuscular e $250 \mu \mathrm{g}$ na submucosa vulvar. Índice superior de 58,6\% de prenhez foi obtido em vacas Shorthorn-Hereford (LAVERDIERE et al., 1995). Sugere-se a indução do estro com análogos de prostaglandina, em vacas que estejam ciclando com bom escore corporal e involução uterina completa no pós-parto.

Com relação ao uso da via submucosa vulvar, vale salientar que o emprego de seringa de $1 \mathrm{~mL}$ e agulha fina $(13 \times 4,5)$ como a utilizada para inocular insulina, facilita a administração do hormônio pois evita refluxo do produto ou lesão local; e não causa reação de defesa física ou movimentos bruscos do animal. Na aplicação do hormônio via intramuscular é recomendável uma boa antissepsia no local da injeção, bem como o uso de agulha 30 x 7, para evitar o refluxo do hormônio.

Sugere-se a realização de novos experimentos com fêmeas zebuínas, visando uma colheita ampla de dados pertinentes ás respostas fisiológicas inerentes ao uso de pequenas doses de prostaglandina e seus análogos.

\section{Conclusão}

Em vacas Nelores, com involução uterina completa e ciclando regularmente, sugere-se a aplicação de cloprostenol para a indução do estro, independentemente da dose e da via de administração, revelando taxas de prenhez similares. Pode-se empregar baixas doses nas duas vias de administração, com menor exposição do animal ao produto e redução do custo do tratamento.

\section{Agradecimentos}

Agradecemos à Fazenda Aurora pelo auxílio financeiro nessa pesquisa.

\section{Referências}

ALVAREZ, R. H.; MEIRELlES, B. C. F. G.; AMBROSANO, A. M. B.; OLIVEIRA, J. V.; POZZIA, J. $\mathrm{R}$. The use of lower doses of the prostaglandin analogue, cloprostenol, of estrus syncronization in heifers. Animal Reproduction Science, Amsterdam, v. 25, n. 2, p. 93-96, 1991.

ALVES, N. G. Estação do ano e tipo de luteólise sobre as características do proestro e estro de vacas das raças Gir e Guzerá, Bos taurus indicus. 1999. Dissertação (Mestrado em Medicina Veterinária) - Curso de Pósgraduação em Medicina Veterinária, Universidade Federal de Minas Gerais, Belo Horizonte.

BANZATTO, D. A.; KRONKA, S. N. Experimentação agrícola. 3. ed. Jaboticabal: FUNEP, 2006.

BO, G. A.; ADAMS, J. P.; PIERSON, R. A.; TRIBULO, H. E.; CACCIA, M.; MAPLETOFT, R. J. Follicular waves dinamics after estradiol-17 $\beta$ treatment of heifers 
with or without a progestogen implant. Theriogenology, Stoneham, v. 41, n. 8, p. 1555-1569, 1994.

BORGES, A. M.; TORRES, C. A. A.; RUAS, J. R. M.; ROCHA JÚNIOR, V. R.; CARVALHO, G. R.; FONSECA, J. F.; MARCATTI NETO, A.; ASSIS, A. J. Características da dinâmica folicular e regressão luteal de vacas das raças Gir e Nelore após tratamento com cloprostenol sódico. Revista Brasileira de Zootecnia, Viçosa, v. 32, n. 1, p. 85-92, 2003.

CASTILHO, C.; DAYAN, A.; BARROS, C. M. Resposta do corpo lúteo de vacas Nelore à $\mathrm{PGF}_{2} \alpha$ administrada por via intramuscular ou submucosa vulvar. Arquivos $d a$ Faculdade de Veterinária, UFRGS, Porto Alegre, v. 25, n. 1, p. 49-60, 1997.

CHACUR, M. G. M.; MENEZES, C. A.; BARBOSA NETTO, L. J.; KRONKA, S. N. Efficiency of half-dosis of cloprostenol subvulvar mucous inducing estrus and pregnancy rate in cows repeat-breeders Nelore-Chianina. Semina, Londrina, v. 26, n. 3, p. 387-394, 2005.

CHACUR, M. G. M.; MIYASAKI, A. A.; PARRON, P. R.; RODELLO, L. Estudo preliminar da sincronização da ovulação em vacas nelore, com acetato de buserelina e D-cloprostenol sódico, cidade do Panamá, Panamá, 2000. In: CONGRESSO PANAMERICANO DE VETERINÁRIA - PANVET, 2000, Cidade do Panamá. Anais...Cidade do Panamá, 2000.

CHAUHAN, F. S.; MGONGO, F. O. K.; KESSY, B. M.; GOMBE, S. Effects of intravulvo-submucosal cloprostenol injections on hormonal profiles and fertility in subestrus cattle. Theriogenology, Stoneham, v. 26, n. 1, p. 69-75, 1986.

COLÉGIOBRASILEIRODEREPRODUÇÃOANIMAL. Normas Técnicas. 1998. Disponível em: <http://www. cbra.org.br/publicacoes/rbra/normastecnicas.doc $>$. Acesso em: 10 mar. 2008.

FERNANDES, C. A. C.; TORRES, C. A. A.; COSTA, E. P. Comparação entre doses e vias de aplicação de cloprostenol para sincronização de estro em bovinos. Revista Brasileira de Reprodução Animal, Belo Horizonte, v. 18, n. 34, p. 105-109, 1994.

FIGUEIREDO, R. A.; BARROS, C. M.; PINHEIRO, O. L.; SOLLER, J. M. P. Ovarian follicular dynamics in Nelore breed (Bos indicus) Cattle. Theriogenology, Stoneham, v. 27, n. 8, p. 1489-5005, 1997.

GALINA, C. S.; ORIHUELA, A.; DUCHATEAU, A. Reproductive physiology in zebu cattle - Unique reproductive aspects that affect their performance. Veterinary Clinics of North American - Food Animal practice, Philadelphia, v. 3, n. 3, p. 6119-632, 1987.
GINTHER, O. J. Local versus systemic utero-ovarian relationships in farm animals. Acta Veterinaria Scandinavica, Copenhagen, v. 77, p. 103-115, 1981.

GINTHER, O. J.; DEL CAMPO, C. H. Vascular anatomy of the uterus and ovaries and the unilateral luteolytic effect of the cattle uterus. American Journal of Veterinary Research, Schaumburg, v. 35, n. 2, p. 193-203, 1974.

HAFEZ, E. S. E.; HAFEZ, B. Reprodução animal, 7. ed. São Paulo: Manole, 2004.

KASTELIC, J. P.; KNOPF. L.; GINTHER, O. J. Effect of day of prostaglandin F-2 $\alpha$ treatment on selection and development of the ovulatory follicle in heifers. Animal Reproduction Science, Amsterdam, v. 23, n. 3, p. 169180, 1990.

KINDAHL, H.; BASU, S.; FREDRIKSSON, G.; GOFF, A.; KUNAVONGKRITK, A.; EDQVIST, L. E. Levels of prostaglandin F-2 $\alpha$ metabolites in blood and urine during early pregnancy. Animal Reproduction Science, Amsterdam, v. 7, p. 133-148, 1984.

LAVERDIERE, G.; ROY, G. L.; PROULX, J.; LAVOIE, D.; DUFOUR, J. J. Estrus synchronization efficiency of PGF-2 $\propto$ injection in Shorthorn-Hereford and crossbred Charolais cattle not having exhibited estrus at 4 or 7 days prior to treatment. Theriogenology, Stoneham, v. 43, n. 5, p. 899-911, 1995.

LOEB, L. The effect of extirpation of the uterus on the life and function of the corpus luteum in the guinea pig. Proceedings of the Society for Experimental Biology and Medicine, Malden, v. 20, p. 441-443, 1923.

LOEB, L. Effects of hysterectomy on system of sex organs and on periodicity of sexual cycle in guinea pigs. American Journal of Physiology, Bethesda, v. 83, p. 202224, 1927.

LUCY, M.C.; SAVIO, J.D.; BADINGA, L.; DELASOTA, R. L.; THATCHER, W. W. Factors that affect ovarian follicular dynamics in cattle. Journal of Animal Science, Champaign, v. 70, n. 11, p. 3615-26, 1992.

LUQUE, E. H.; HUTTER, J. C.; MONTES, G. S. Regulacion hormonal de los ciclos reproductivos em la vaca. Revista de Medicina Veterinaria, São Paulo, v. 64, n. 3, p. 190-211, 1983.

MORENO, I. Y. D.; GALINA, C. S.; ESCOBAR, F. J.; RAMIREZ, B.; NAVARRO FIERRO, R. Evaluation of the lytic responce of prostaglandin F-2 alpha in zebu cattle based on serum progesterone. Theriogenology, Stoneham, v. 25, n. 3, p. 413-421, 1986. 
ONO, H.; TERAWAKY, Y.; OHBOSHI, K.; YAMAZAKI, D. An intravulvosubmucous injection PGF-2 $\propto$ in anestrus cows. Animal Reproduction Science, Amsterdam, v. 5, n. 1, p. 1-5, 1982.

PESSÔA, V. M. Avaliação da eficiência de baixas doses de prostaglandina na indução da luteólise e do estro em vacas Nelore. 2003. Dissertação (Mestrado em Medicina Veterinária) - Curso de Pós-graduação em Medicina Veterinária, Universidade Estadual Paulista, Botucatu.

PINHEIRO, O. L.; BARROS, C. M.; FIGUEIREDO, R. A.; VALLE, E. R.; ENCARNAÇÃO, R. O.; PADOVANI, C. R. Estrous behaviour and the estrus-to-ovulation interval in Nelore cattle (Bos indicus) with natural estrus or estrus induced with prostaglandin F2 $\alpha$ or Norgestomet and estradiol valerate. Theriogenology, Stoneham, v. 49, n. 3, p. 667-681, 1998.

RAdOSTITIS, O. M.; BLOOD, D. C. Manual de controle da saúde e produção de animais. São Paulo: Manole, 1986. 189 p.
SAVIO, J. D.; BOLAND, M. P.; ROCHE, J. F. Development of dominant follicles and length of ovarian cycles in post-partum dairy cows. Journal of Reproduction and Fertility, Cambridge, v. 88, n. 2, p. 581-591, 1990.

SCHALLER, O. Nomenclatura anatômica veterinária ilustrada. Zaragoza: Acribia, 1993. p. 213-223.

TANABE, T. Y.; HANN, R. C. Synchronized estrus and subsequent conception in dairy heifers treated with prostaglandin F-2 $\propto$ influence of stage of the cycle at treatment. Journal of Animal Science, Champaign, v. 58, n. 4, p. 805-811, 1984.

VALLE, E. R. O ciclo estral de bovinos e métodos de controle. Campo Grande: EMBRAPA, 1991.

WRIGHT, P. J.; MALMO, J. Pharmacological manipulacion of fertility. The Veterinary Clinics of North America, Philadelphia, v. 8, p. 57-89, 1992. 